Research Paper

\title{
A Novel Pre-treatment Model Predicting Risk of Developing Refractoriness to Transarterial Chemoembolization in Unresectable Hepatocellular Carcinoma
}

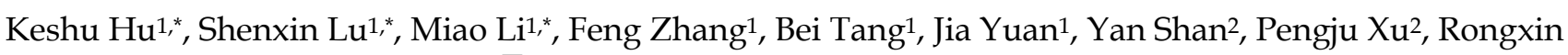
Chen ${ }^{1}$, Zhenggang Ren ${ }^{1}$, Xin Yin ${ }^{1 凶}$

1. Liver Cancer Institute \& Zhongshan Hospital, Fudan University, Shanghai, China; Key Laboratory of Carcinogenesis and Cancer Invasion, Ministry of Education

2. Department of Radiology, Zhongshan Hospital, Fudan University, Shanghai, China

*These authors contributed equally to this paper.

$\triangle$ Corresponding author: Xin Yin, Liver Cancer Institute \& Zhongshan Hospital, Fudan University, 136 Yi Xue Yuan Road, Shanghai 200032, China. E-mail: yin.xin@zs-hospital.sh.cn; Tel: +86-21-64038038

(C) The author(s). This is an open access article distributed under the terms of the Creative Commons Attribution License (https://creativecommons.org/licenses/by/4.0/). See http://ivyspring.com/terms for full terms and conditions.

Received: 2020.02.12; Accepted: 2020.05.02; Published: 2020.05.18

\begin{abstract}
Background and aim: Refractoriness to transarterial chemoembolization is common during the therapeutic process of hepatocellular carcinoma, which is an intractable issue and may compromise the prognosis. We aim to establish a pre-treatment model to identify patients with high risks of refractoriness.

Methods: From 2010 to 2016, 824 treatment-naive patients who had initially underwent at least two sessions of transarterial chemoembolization in Zhongshan Hospital, Fudan University were retrospectively enrolled. These patients were randomly allocated into a training cohort and a validation cohort. The pre-treatment scoring model was established based on the clinical and radiological variables using logistic regression and nomogram. The discrimination and calibration of the model were also evaluated.

Results: Logistic regression identified vascularization pattern, ALBI grade, serum alpha-fetoprotein level, serum Y-glutamyl transpeptidase level and major tumor size as the key parameters related to refractoriness. The P-TACE model was established using these variables (risk score range: $0-19.5$ ). Patients were divided into six risk subgroups based on their scores $(<4, \geq 4, \geq 7, \geq 10, \geq 13, \geq 16)$. The discriminative ability, as determined by the area under receiver operating characteristic curve was 0.784 (95\% confidence interval: $0.741-0.827)$ in the training cohort and 0.743 ( $95 \%$ confidence interval: $0.696-0.789$ ) in the validation cohort. Moreover, satisfactory calibration was confirmed by Hosmer-Lemeshow test with $P$ values of 0.767 and 0.913 in the training cohort and validation cohort.

Conclusions: This study presents a pre-treatment model to identify patients with high risks of refractoriness after transarterial chemoembolization and shed light on clinical decision making.
\end{abstract}

Key words: Hepatocellular carcinoma, Transarterial chemoembolization, Refractoriness, Risk stratification

\section{Introduction}

Hepatocellular carcinoma (HCC) is the sixth most common malignancy and the second leading cause of cancer-related death worldwide [1]. According to a recent global view of HCC [2], almost
$85 \%$ of HCC are estimated to occur in the developing area, particularly in Eastern Asia and sub-Saharan Africa. Besides, the HCC population in China, which are mainly ascribed to chronic hepatitis B virus (HBV) 
infection, are supposed to have an earlier onset of a mean age of 52 years old, and more than $60 \%$ of such patients present with intermediate-stage or advanced-stage HCC when diagnosed[2]. Transarterial chemoembolization (TACE) is currently the recommended treatment option for intermediate stage HCC with well-preserved liver function and performance status [3]. TACE benefits patients in two ways: providing a treatment response and minimizing liver function damage by infusion embolization and chemotherapeutic agents into tumor feeding arteries [4]. Nevertheless, in some cases, despite an initial response induced by TACE, repetitive TACE treatments could impair liver function gradually and induce stenosis of the hepatic artery. Even worse, TACE is associated with disturbances of tumor microenvironment, which results in hypoxia and upregulation of vascular endothelial growth factor (VEGF), thus promotes tumor invasion and metastasis [5]. As such, with the increased sessions of TACE procedure, the treatment efficacy diminishes and patients then probably enter a state of so-called "TACE refractoriness".

The concept of TACE refractoriness was first proposed by the Japan Society of Hepatology and updated as JSH-LCSGJ Criteria in 2014[4, 6]. According to these criteria, TACE-refractory HCC patients who belong to Child-Pugh grade $\mathrm{A}$ are candidates for molecular target therapy as a second-line treatment option [7]. However, for patients with deteriorated liver function induced by repetitive TACE treatments, few subsequent treatment options are available and the prognosis is usually dismal $[8,9]$. If TACE refractoriness could be identified earlier, with compensated liver function, these patients could switch to molecular target therapy or other systematic treatments, avoiding exposure to the adverse effects of TACE. Actually, the necessity of early identification of TACE refractoriness in HCC population undergoing TACE treatments has been proposed by recent EASL guidelines [10].

Of note, the range of indications for TACE covers a wide spectrum in real-world clinical practice. Besides intermediate stage HCC, early stage HCC that deemed as ineligible for curative treatments due to significant liver impairment or general contraindication is also recommended as TACE candidates, according to the treatment stage migration principle [11]. These patients are quite heterogeneous with different degrees of liver dysfunction and tumor burden, leading to a variable median overall survival of 13-43 months [12]. Furthermore, treatment decisions in the individual patient are partially subjective, being largely dependent on the physician's decision within each institution. Development of an individual prediction model could allow for objective selection of ideal TACE candidates and guide decision for treatments. Indeed, there are several existing prognostic models such as hepatoma arterial-embolization prognostic (HAP) score [13], mHAP-II score[14], the Assessment for Retreatment with TACE (ART) score[15], etc. These existing prognostic models, however, have been questioned due to being incapable to predict patient outcome accurately in independent validation cohorts, causing doubt upon their clinical utility[16, 17]. Till now, no pre-treatment model evaluating the risk of TACE refractoriness has been established. Herein, we developed a novel scoring model based on pre-treatment clinical and radiological parameters for the early identification of TACE-refractoriness in early/intermediate stage HCC following TACE treatment.

\section{Methods}

\section{Patients}

From 2010 to 2016, patients who were diagnosed with unresectable HCC and received TACE treatment at liver cancer institute, Zhongshan hospital were screened. The unresectable HCC was defined according to the comprehensive assessment of the size, number, location of the tumors as well as the preserved liver function of the patient. Patients were included who met the following criteria: 1) with HCC diagnosis confirmed by pathological or clinical diagnosis according to the AASLD criteria [18]; 2) with BCLC stage A/B stage HCC that had undergone at least two sessions of TACE treatment; 3) with Child-Pugh grade of A or B before treatment; 4) with Eastern Cooperative Oncology Group performance status score of $0-1$. Patients were excluded if they: 1 ) had received other anti-tumor treatments prior to TACE; 2) with serious dysfunction of the heart or kidney; 3) with other malignancies in addition to HCC. Patients were 1:1 assigned to training cohort and validation cohort by the random numbers generated by the software Stata. This study was approved by the institutional review board of Zhongshan Hospital and complied with the standards of the Declaration of Helsinki and current ethical guidelines.

\section{TACE procedure, follow up and evaluation of TACE refractoriness}

TACE was performed by the same team of hepatologists with more than 10-year experience, under the institutional standard protocol as described by Yin et al. [19]. Briefly, a 5F or 4F catheter was 
introduced into the abdominal aorta under fluoroscopy guidance. Angiography was performed to identify the tumors and their feeding hepatic arteries. Anticancer drugs (1000 mg of 5-fluorouracil, $100 \sim 150 \mathrm{mg}$ of oxaliplatin) were infused into the tumor feeding arteries. Super-selective embolization was performed by using a microcatheter if needed. An emulsion of 5 to $20 \mathrm{~mL}$ lipiodol with $30 \mathrm{mg}$ epirubicin was slowly injected into the feeding arteries.

Four weeks after treatment, patients were followed by computed tomography (CT) and/or magnetic resonance imaging (MRI), liver function, blood routine test and tumor markers. The effects of TACE were assessed by using dynamic CT/MRI and residual enhancement of nodules was measured with consideration of the modified response evaluation criteria in solid tumors (mRECIST). If no residual viable tumors were indicated based on imaging results, patients were followed up every 2 or 3 months for one year and every six months thereafter. If residual viable or newly developed tumors were identified, repeated TACE were performed on an "on-demand" basis depending on individual tumor response and hepatic functional reserve [20].

TACE refractoriness was judged by the our hepatologists according to JSH-LCSGJ Criteria: two or more consecutive insufficient responses of the treated tumor (viable lesion $>50 \%$ ) or increases of tumor number after changing the chemotherapeutic agents and/or reanalysis of the feeding artery seen on response evaluation $\mathrm{CT} / \mathrm{MRI}$ at 1-3 months after having adequately performed selective TACE; the appearance of vascular invasion; the appearance of extrahepatic spread; a continuous elevation of tumor markers immediately after TACE even though slight transient decrease is observed[4].

\section{Clinical and radiological variables contributing to develop prediction model}

To evaluate the potential predicting variables, the clinical variables included the patient's age, sex, etiology, laboratory tests, tumor biomarkers, tumor size, tumor number and radiological features. The ALBI score was calculated and graded as indicated by previous studies [21]. A preoperative neutrophil-tolymphocyte ratio (NLR) was also calculated within 7 days before TACE. Additionally, the radiological features, especially the enhancement characteristics of the main target tumors were determined based on four-fold categorization of HCC vascularization patterns [22, 23] on baseline CT/MRI. Two experienced radiologists who did not know baseline clinical data assessed overall tumor response and vascularization patterns. In detail, Type-1 represented a "homogeneous enhancement" pattern with no increase in arterial blood flow. The Type-2 pattern represented "homogeneous enhancement" pattern with increased arterial blood flow. Type-3 is a heterogeneous enhancement pattern with a septum-like structure; and Type- 4 is an irregularly shaped ring structure enhancement pattern. Typical images of vascularization pattern were illustrated in Figure S1. These clinical and laboratory variables were measured before TACE therapy and were collected from patients' records.

\section{Statistical analysis}

This analysis was reported according to the TRIPOD (Transparent reporting of a multivariable prediction model for individual prognosis or diagnosis) guidelines [24]. Continuous variables were presented as an average \pm standard deviation or as the median and its range. The normal distribution of the continuous variables were tested by Shapiro-Wilk tests, and the subsequent hypothesis tests were performed by either student t-test for normal distributed variables, or rank-sum (Mann-Whitney) test for abnormal distributed variables. Categorical variables were tested by Fisher exact test. The risk factors related to TACE refractoriness were evaluated by logistic regression, and all the variables except for albumin, bilirubin and Child-Pugh grade were included in the multivariate regression, in case of the collinearity. The cut-off levels of the specific factors were determined by receiver operating characteristic curve (ROC). The development of the pre-treatment scoring model was also based on the logistic regression of multiple variables, which was illustrated as a nomogram as well. The discrimination efficacy of the model was examined by the area under ROC (AUROC), and the goodness of fit were validated by calibration curve and Hosmer-Lemeshow $(\mathrm{H}-\mathrm{L})$ test, in which case $\mathrm{P}$ value $>0.05$ indicated good performance. All the statistical analyses were conducted by software Stata 14.0 for Windows (StataCorp, College Station, TX) or R language (version 3.5.2; R Package for Statistical Computing; www.r-project.org). $\mathrm{P}<0.05$ was considered statistically significant.

\section{Results}

\section{Clinicopathological characteristics}

From January 2010 to December 2016, a total of 1661 patients were screened and 824 eligible patients were enrolled and randomly assigned to training cohort $(n=412)$ and validation cohort $(n=412)$. Patient characteristics are summarized in Table 1 . The clinicopathological characteristics of the patients does not differ significantly between the training cohort 
and validation cohort (All P > 0.05). Generally, most of the patients were male $(n=698,84.7 \%)$ and with HBV infection $(n=654,79.4 \%)$. The patients enrolled in the present study had tumors with the median major size of $6.0 \mathrm{~cm}$ (range: $0.5-21.5 \mathrm{~cm}$ ) and the median number of 1 (range: 1-9), of whom 57.2\% $(n=471)$ patients had a single tumor. The majority of the patients were diagnosed as BCLC stage A $(n=502$, $60.9 \%$ ) with a favorable liver function of Child-Pugh grade $\mathrm{A}(\mathrm{n}=804,97.6 \%)$. The median TACE times that all the patients had undergone were 3 times (range: $2-16)$, and approximately half $(n=406,49.3 \%)$ of the patients eventually developed TACE refractoriness judged by JSH-LCSGJ Criteria [4].

Table 1. The baseline characteristics of the patients in training cohort $(n=412)$ and validation cohort $(n=412)$

\begin{tabular}{|c|c|c|c|c|}
\hline & $\begin{array}{l}\text { Total } \\
(\mathrm{n}=824)\end{array}$ & $\begin{array}{l}\text { Training } \\
\text { Cohort } \\
(n=412)\end{array}$ & $\begin{array}{l}\text { Validation } \\
\text { Cohort } \\
(\mathrm{n}=412)\end{array}$ & \\
\hline Gender, (male/female) & $698 / 126$ & $345 / 67$ & $353 / 59$ & $\mathrm{P}=0.498$ \\
\hline Age, years & $59.2 \pm 11.8$ & $59.2 \pm 11.7$ & $59.3 \pm 11.9$ & $\mathrm{P}=0.920$ \\
\hline HBV infection, (no/yes) & $170 / 654$ & $79 / 333$ & $91 / 321$ & $\mathrm{P}=0.344$ \\
\hline BCLC stage, (A/B) & $502 / 322$ & $245 / 167$ & $257 / 155$ & $\mathrm{P}=0.432$ \\
\hline Serum total bilirubin, $\mu \mathrm{mol} / \mathrm{L}$ & $13.9 \pm 7.8$ & $13.7 \pm 7.7$ & $14.1 \pm 7.8$ & $\mathrm{P}=0.331$ \\
\hline Serum $\gamma$-GT, U/L & $\begin{array}{l}95[12 \sim \\
998]\end{array}$ & 94 [12 998] & $98[16 \sim 827]$ & $\mathrm{P}=0.356$ \\
\hline Serum albumint, $\mathrm{g} / \mathrm{L}$ & $38.3 \pm 5.3$ & $38.1 \pm 5.2$ & $38.4 \pm 5.5$ & $\mathrm{P}=0.399$ \\
\hline $\begin{array}{l}\text { Serum AFP, ng/ml } \\
\text { (median[range]) }\end{array}$ & $\begin{array}{l}107[1 \sim \\
>60500]\end{array}$ & $\begin{array}{l}125.5[1 \sim \\
>60500]\end{array}$ & $\begin{array}{l}99.5[1 \sim \\
>60500]\end{array}$ & $\mathrm{P}=0.219$ \\
\hline Child-Pugh grade, (A/B) & $804 / 20$ & $400 / 12$ & $404 / 8$ & $\mathrm{P}=0.498$ \\
\hline $\begin{array}{l}\text { Neutrophil-to-lymphocyte } \\
\text { ratio, (median[range]) }\end{array}$ & $\begin{array}{l}3[0.5 \sim \\
86.7]\end{array}$ & $2.9[0.7 \sim 53.1]$ & $3.1[0.5 \sim 86.7]$ & $\mathrm{P}=0.133$ \\
\hline $\begin{array}{l}\text { Major tumor size group, } \\
(\leq 5 \mathrm{~cm} />5 \mathrm{~cm})\end{array}$ & $355 / 469$ & $186 / 226$ & $169 / 243$ & $\mathrm{P}=0.260$ \\
\hline $\begin{array}{l}\text { Number of tumors, } \\
\text { (single/multiple) }\end{array}$ & $471 / 353$ & $227 / 185$ & $244 / 168$ & $\mathrm{P}=0.260$ \\
\hline $\begin{array}{l}\text { Vascularization patterns, (type } \\
1 \& 2 \text { / type } 3 \& 4 \text { ) }\end{array}$ & $455 / 369$ & $222 / 190$ & $233 / 179$ & $\mathrm{P}=0.484$ \\
\hline ALBI grade ${ }^{\dagger},(1 / 2 / 3)$ & $399 / 415 / 9$ & $195 / 212 / 5$ & $204 / 203 / 4$ & $\mathrm{P}=0.778$ \\
\hline TACE times, (median[range]) & $3[2 \sim 16]$ & $3[2 \sim 13]$ & $4[2 \sim 16]$ & $\mathrm{P}=0.233$ \\
\hline TACE refractoriness, (no/yes) & $418 / 406$ & $210 / 202$ & $208 / 204$ & $\mathrm{P}=0.944$ \\
\hline
\end{tabular}

†1 value for albumin and ALBI grade were missing

HBV, hepatitis B virus; BCLC stage, Barcelona Clinic Liver Cancer stage; $\gamma_{-G T}$, $\mathrm{Y}$-glutamyl transpeptidase; AFP: alpha-fetoprotein; ALBI grade: albumin-bilirubin grade; TACE: transarterial chemoembolization

\section{Risk factors and cut-offs}

All the potential risk factors were summarized in Table 2 and univariate logistic regression was performed in order to calculate the unadjusted odds ratio (OR). Except for the ALBI grade, the other associated factors including serum total bilirubin, serum albumin and Child-Pugh grade were excluded from the further multivariate regression ascribed to the collinearity. ALBI grade was retained referring to the previous study [25]. As a result, the vascularization pattern, ALBI grade and major tumor size demonstrated obvious significance in TACE refractoriness (Table 2). Considering that the serum alpha-fetoprotein (AFP) level and $\gamma$-glutamyl transpeptidase $(\gamma-G T)$ played an important role in clinical practice, and statistical differences were observed in univariate regression, eventually they were employed for the establishment of the model as well, together with the vascularization pattern, ALBI grade and major tumor size.

Table 2. The respective OR of the possible risk factors related to TACE refractoriness

\begin{tabular}{|c|c|c|c|c|}
\hline Risk & $\begin{array}{l}\text { Unadjusted OR } \\
{[95 \% \mathrm{CI}]}\end{array}$ & $\begin{array}{l}P \\
\text { value }\end{array}$ & $\begin{array}{l}\text { Adjusted OR [95\% } \\
\mathrm{CI}]^{\ddagger}\end{array}$ & $\begin{array}{l}\mathrm{P} \\
\text { value }\end{array}$ \\
\hline Gender, (male/female) & $1.306[0.891-1.913]$ & 0.171 & $1.451[0.872-2.416]$ & 0.152 \\
\hline Age, years & $0.993[0.982-1.005]$ & 0.250 & $0.997[0.980-1.015]$ & 0.765 \\
\hline HBV infection, (no/yes) & $1.421[1.011-1.997]$ & $0.043^{*}$ & $1.553[0.938-2.574]$ & 0.087 \\
\hline BCLC stage, (A/B) & $1.049[0.793-1.388]$ & 0.738 & 595-1.888] & 0.843 \\
\hline $\begin{array}{l}\text { Serum total bilirubin, } \\
\mu \mathrm{mol} / \mathrm{L}\end{array}$ & $1.021[1.003-1.040]$ & $0.021^{*}$ & NA & - \\
\hline Serum $\gamma$-GT, U/L & $1.003[1.002-1.004]$ & $<0.001^{*}$ & $1.001[0.999$ & 0.363 \\
\hline Serum albumin, $\mathrm{g} / \mathrm{L}$ & $0.429[0.322-0.572]$ & $<0.001^{*}$ & NA & - \\
\hline $\mathrm{P}, \mathrm{ng} / \mathrm{ml}$ & $-1.000]^{\dagger}$ & $<0.001^{*}$ & $1.000[1.000-1.000]^{\dagger}$ & 0.089 \\
\hline Child-Pugh grade, (A/B) & $0.839[0.344-2.046]$ & 0.699 & NA & - \\
\hline $\begin{array}{l}\text { Neutrophil-to-lymphocyte } \\
\text { ratio }\end{array}$ & 1.007[0.975-1.040] & 0.671 & $1.015[0$ & 0.412 \\
\hline Major tumor size, $\mathrm{cm}$ & $1.124[1.078-1.172]$ & $<0.001^{*}$ & $1.106[1.035-1.182]$ & $0.003^{*}$ \\
\hline Number of tumors & $0.998[0.913-1.092]$ & 0.968 & $1.003[0.838-1.201]$ & 0.971 \\
\hline $\begin{array}{l}\text { Vascularization pattern, } \\
\text { (type } 1 \& 2 / \text { type } 3 \& 4 \text { ) }\end{array}$ & $3.769[2.820-5.038]$ & $<0.001^{*}$ & $3.473[2.354-5.124]$ & $<0.001^{*}$ \\
\hline ALBI grade, $(1 / 2 \& 3)$ & $2.608[1.983-3.431]$ & $<0.001^{*}$ & $2.379[1.648-3.434]$ & $<0.001^{*}$ \\
\hline \multicolumn{5}{|c|}{$\begin{array}{l}\text { ‡The parameters serum total bilirubin, serum albumin and Child-Pugh grade were } \\
\text { excluded from the multivariate logistic regression due to the collinearity with ALBI } \\
\text { grade. }\end{array}$} \\
\hline \multicolumn{5}{|c|}{$\begin{array}{l}\text { OR, odds ratio; CI, confidence interval; HBV, hepatitis B virus; BCLC stage, } \\
\text { Barcelona Clinic Liver Cancer stage; } \gamma-G T \text {, } \gamma \text {-glutamyl transpeptidase; AFP: } \\
\text { alpha-fetoprotein; ALBI grade: albumin-bilirubin grade; NA, not applicable }\end{array}$} \\
\hline
\end{tabular}

AUROC was calculated to determine the optimal cut-off values of AFP, $\gamma$-GT and the major tumor size, to establish an easy-to-use prediction model and simultaneously maintain its discrimination efficacy. The ideal single cut-off level of the three values calculated by Youden index were $78 \mathrm{ng} / \mathrm{ml}$ (Youden index=0.224), 69.5U/L (Youden index $=0.218$ ) and $6.1 \mathrm{~cm}$ (Youden index=0.198). Afterwards, slight adjustments were made to maintain the accuracy and for a convenient usage. Respectively, the cut-offs were $80 \mathrm{ng} / \mathrm{mL}, 4000 \mathrm{ng} / \mathrm{mL}$ for AFP; 50U/L, 75U/L, 135U/L for $\gamma$-GT; and $5 \mathrm{~cm}$ for major tumor size (Table S1). The Youden indexes were $0.217(80 \mathrm{ng} / \mathrm{mL}), 0.153$ (4000ng/mL), 0.159 (50U/L), 0.210 (75U/L), 0.175 $(135 \mathrm{U} / \mathrm{L})$, and $0.184(5 \mathrm{~cm})$ for the referred parameters, respectively. The ROC curves were shown in Figure S2.

\section{Development of the p-TACE model}

Logistic regression of the risk factors for TACE refractoriness, including vascularization pattern, ALBI grade, AFP grade, $\gamma$-GT grade and major tumor size, was performed in the training cohort $(n=412)$. The results were summarized in Table 3 and a nomogram was illustrated in Figure 1. For a convenient but accurate prediction, the scoring model 
was further developed on the basis of the logistic regression and nomogram. Accordingly, the model sum the respective score of five factors, as described in Table 4. Furthermore, the prediction efficacy was evaluated by the comparison between the p-TACE model vs. the logistic regression model in AUROC ( 0.784 vs. 0.787$)$ and $\mathrm{H}-\mathrm{L}$ test $(\mathrm{P}=0.767$ vs. $\mathrm{P}=0.748)$, suggesting a satisfactory maintenance of prediction efficacy in the new scoring model.

Table 3. The results of logistic regression in training cohort $(n=412)$

\begin{tabular}{|c|c|c|c|}
\hline Risk factors & Coefficient & OR $[95 \% \mathrm{CI}]$ & $P$ value \\
\hline $\begin{array}{l}\text { Vascularization pattern (Type } \\
3 / 4 \text { vs. Type } 1 / 2 \text { ) }\end{array}$ & 1.327 & $3.769[2.403-5.911]$ & $<0.001^{*}$ \\
\hline $\begin{array}{l}\text { ALBI grade (Grade } 2 / 3 \text { vs. } \\
\text { Grade } 1 \text { ) }\end{array}$ & 0.936 & $2.551[1.625-4.004]$ & $<0.001^{*}$ \\
\hline $\begin{array}{l}\text { Major tumor size ( }>5 \mathrm{~cm} \text { vs. } \\
\leq 5 \mathrm{~cm})\end{array}$ & 0.531 & $1.700[1.050-2.752]$ & $0.031^{*}$ \\
\hline \multicolumn{4}{|l|}{ AFP grade } \\
\hline Grade 2 vs. Grade 1 & 0.783 & $2.188[1.331-3.597]$ & $0.002^{*}$ \\
\hline Grade 3 vs. Grade 1 & 1.217 & $3.377[1.779-6.413]$ & $<0.001^{*}$ \\
\hline \multicolumn{4}{|l|}{$\gamma$-GT grade } \\
\hline Grade 2 vs. Grade 1 & 0.259 & $1.296[0.629-2.671]$ & 0.482 \\
\hline Grade 3 vs. Grade 1 & 0.526 & $1.692[0.873-3.277]$ & 0.119 \\
\hline Grade 4 vs. Grade 1 & 0.935 & $2.547[1.310-4.952]$ & $0.006^{*}$ \\
\hline Constant & -5.242 & - & - \\
\hline
\end{tabular}

\section{Validation of the P-TACE model}

The prediction efficacy of p-TACE scoring model was further evaluated in the validation cohort $(n=412)$ as well. Consistent results were obtained in both training cohort and validation cohort, including AUROC (0.784 [95\% CI: 0.741-0.827] vs. 0.743 [95\% CI: $0.696-0.789])$, H-L test $(\mathrm{P}=0.767$ vs. 0.913), risk stratification (Table 5) and calibration curves (Figure 2). All these results confirmed the favorable predictive capacity of the novel model in identifying the risk of TACE-refractoriness prior to TACE treatment.

Table 4. The novel scoring model ( $p$-TACE model) for pre-treatment prediction of TACE refractoriness

\begin{tabular}{|c|c|c|c|c|}
\hline Risk factors & Grade & Score & & \\
\hline \multirow[t]{2}{*}{$\begin{array}{l}\text { Vascularization } \\
\text { pattern }\end{array}$} & $\begin{array}{l}\text { Type } \\
1 / 2\end{array}$ & 0 & & \\
\hline & $\begin{array}{l}\text { Type } \\
3 / 4\end{array}$ & 5.5 & & \\
\hline \multirow[t]{2}{*}{ ALBI grade } & 1 & 0 & & \\
\hline & $2 / 3$ & 3.5 & & \\
\hline \multirow[t]{3}{*}{ AFP level, ng/mL } & $\sim 80$ & 0 & & \\
\hline & $\sim 4000$ & 3 & & \\
\hline & $\begin{array}{l}>4000 \\
\sim 50\end{array}$ & $\begin{array}{l}5 \\
0\end{array}$ & $\begin{array}{l}\text { Scoring model } \\
\text { grade (score) }\end{array}$ & $\begin{array}{l}\text { Estimated risk of TACE } \\
\text { refractoriness }\end{array}$ \\
\hline \multirow{3}{*}{ Y-GT level, U/L } & $\sim 75$ & 1 & Grade $1(<4)$ & $15 \%$ or less \\
\hline & $\sim 135$ & 2 & Grade $2(\geq 4)$ & appr. $30 \%$ \\
\hline & $>135$ & 3.5 & Grade $3(\geq 7)$ & appr. $40 \%$ \\
\hline \multirow{2}{*}{$\begin{array}{l}\text { Major tumor size, } \\
\mathrm{cm}\end{array}$} & $\sim 5$ & 0 & Grade $4(\geq 10)$ & appr. $60 \%$ \\
\hline & $>5$ & 2 & Grade $5(\geq 13)$ & appr. $75 \%$ \\
\hline Total & & $\begin{array}{l}\text { range } 0 \\
\sim 19.5\end{array}$ & Grade $6(\geq 16)$ & $85 \%$ or more \\
\hline
\end{tabular}

Points

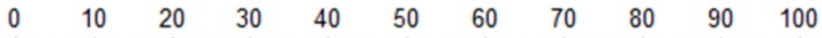

Points

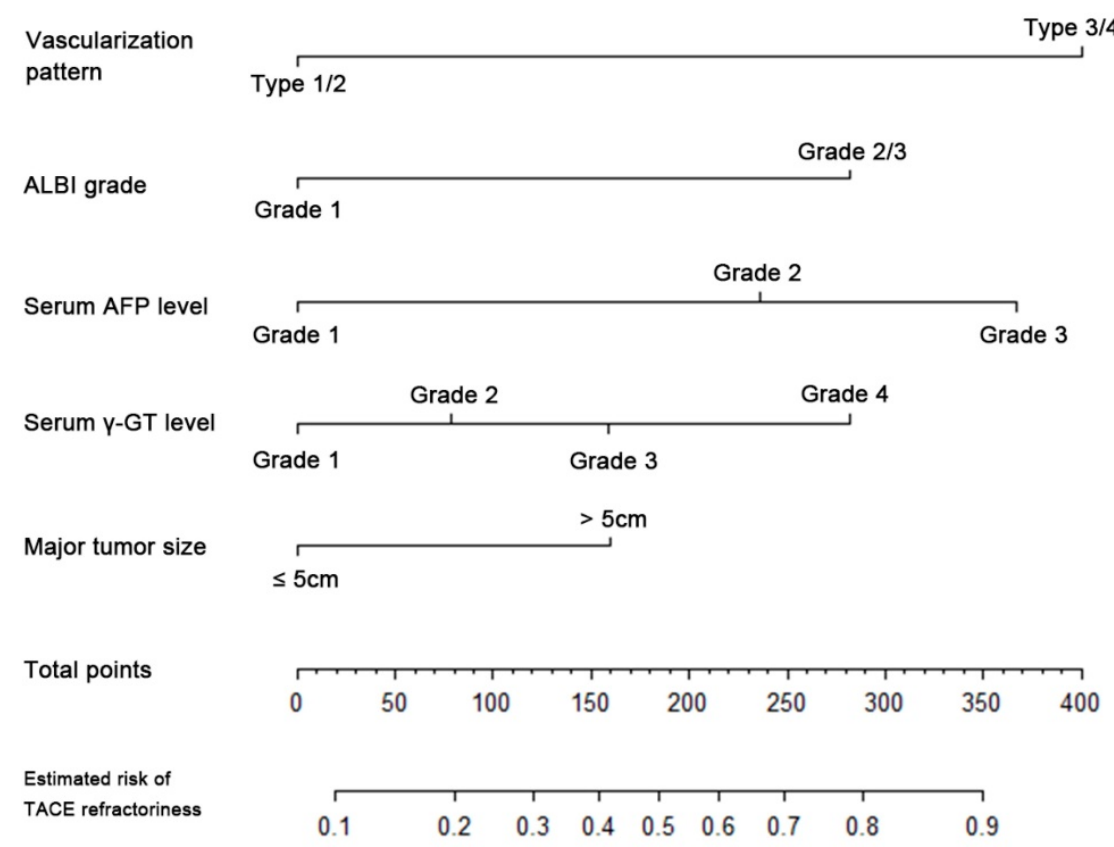

Figure 1. The nomogram of logistic regression model for TACE refractoriness in training cohort. TACE, transarterial chemoembolization; ALBI grade, albumin-bilirubin grade; AFP, alpha-fetoprotein; Y-GT, Y-glutamyl transpeptidase 
(a)

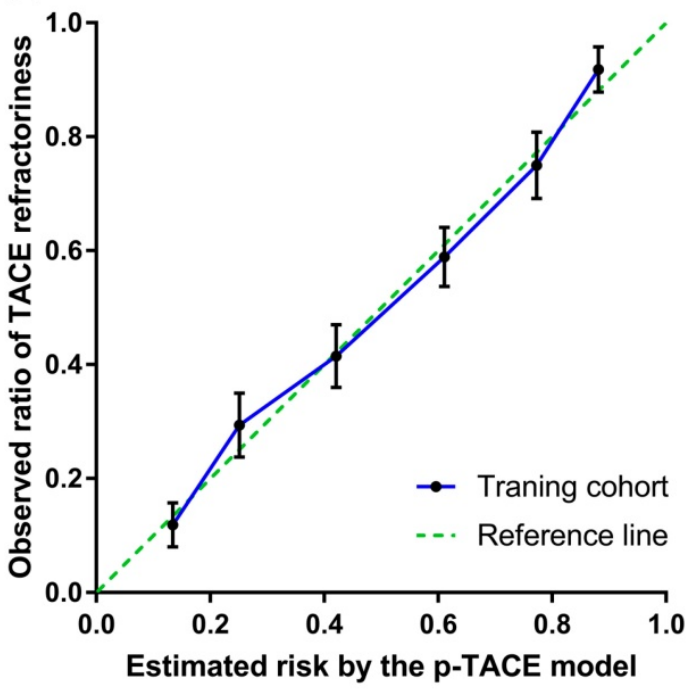

(b)

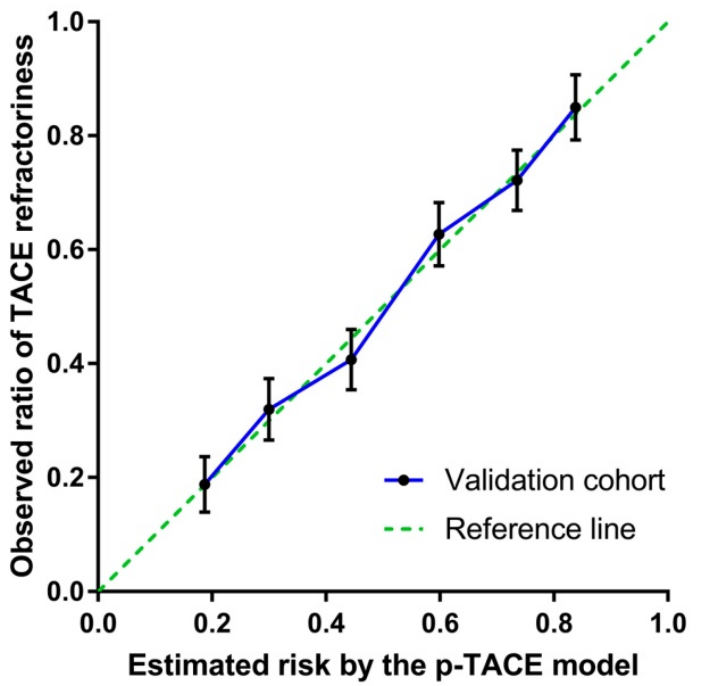

Figure 2. The calibration curve of the p-TACE model in training and validation cohort. (a) the calibration curve of training cohort; (b) the calibration cure of validation cohort. TACE, transarterial chemoembolization

Table 5. The estimated and observed risks of TACE-refractoriness by the P-TACE model in training and validation cohort

\begin{tabular}{lllll}
\hline $\begin{array}{l}\text { Scoring } \\
\text { model }\end{array}$ & \multicolumn{2}{l}{ Training cohort $(\mathrm{n}=412)$} & \multicolumn{2}{l}{ Validation cohort $(\mathrm{n}=412)$} \\
\cline { 2 - 5 } & $\begin{array}{l}\text { Estimated } \\
\text { risk }(\%)\end{array}$ & $\begin{array}{l}\text { Observed ratio }[95 \% \\
\mathrm{CI}](\%)\end{array}$ & $\begin{array}{l}\text { Estimated Observed ratio }[95 \% \\
\text { risk }(\%)\end{array}$ & $\mathrm{CI}](\%)$ \\
\hline Grade 1 & 13.4 & $11.9[6.0-22.2]$ & 18.7 & $18.8[10.9-30.3]$ \\
Grade 2 & 25.1 & $29.4[19.8-41.4]$ & 30.0 & $32.0[22.4-43.4]$ \\
Grade 3 & 42.1 & $41.5[31.3-52.4]$ & 44.4 & $40.7[30.8-51.4]$ \\
Grade 4 & 61.1 & $58.9[48.4-68.6]$ & 59.8 & $62.7[51.2-72.9]$ \\
Grade 5 & 77.3 & $75.0[61.9-84.7]$ & 73.5 & $72.2[60.7-81.4]$ \\
Grade 6 & 88.1 & $91.8[80.0-96.9]$ & 83.8 & $85.0[70.1-93.2]$ \\
\hline
\end{tabular}

CI: confidence interval

\section{Discussion}

In present study, we established a pre-treatment scoring model (p-TACE model) based on five common clinical and radiological variables. The p-TACE model successfully stratified patients according to their risks of developing TACE refractoriness. With good discrimination efficacy and calibration, this easy-to-use model facilitates the pre-treatment individualized prediction of TACE refractoriness and contributes to clinical decision making.

Our model was developed based on the vascularization pattern, ALBI grade, AFP level, $\gamma$-GT level and major tumor size, which were significant factors associated with TACE refractoriness in logistic regression model. To be specific, the vascularization pattern and the major tumor size or tumor burden affects the treatment efficacy of TACE; ALBI grade is an assessment of liver function reserve and associated with HCC prognosis [26]; AFP level is related to the differentiation of the cancer [27]; and $\gamma$-GT level has been reported to be associated with tumor relapse and poor survival outcome [28]. These synergistic effects provided a rationale for the prediction efficacy of the novel p-TACE scoring model.

Compared to the nomogram, the p-TACE scoring model is more convenient for clinical usage, with an equal prediction efficacy proved by AUROC and H-L test. According to our model, patients with a high risk of developing TACE refractory status (eg, $>50 \%$ ) should not receive repetitive TACE. Treatment migration strategy such as molecular target therapy, immunotherapy should be considered, since they were probably TACE non-responders and would not benefit from TACE monotherapy. In accordance, the OPTIMIS study revealed that continuing TACE after TACE refractoriness would lead to deterioration of liver function and unfavorable prognosis [29]. More recently, the results from a randomized, multicenter, phase II clinical trial (TACTICS trial) indicated that TACE plus Sorafenib significantly improved progression-free survival over TACE alone in patients with intermediate HCC [30]. The duration time of Sorafenib administration was positively associated with progression-free survival outcome. Thus, early introduction of effective systematic treatment to patients with high risk of TACE refractoriness could improve prognosis in comparison with TACE monotherapy. This treatment migration concept is supported by the ESMO clinical practice guideline for HCC [31].

The p-TACE model is established to predict the risk of TACE refractoriness prior to TACE treatment. In the model, we employed an important factor, the homogeneous/heterogeneous vascularization of the tumor. Several lines of studies indicated the post-treatment lipiodol deposition and the response 
to TACE were correlated with tumor vascularization $[32,33]$. Besides, it has been reported that heterogeneous vascularization within the tumor is associated with poorly differentiated HCC cells [34]. After repetitive TACE treatment, these poorly differentiated tumor cells are probably empowered with stemness properties and chemoresistance under hypoxia microenvironment induced by TACE $[35,36]$. Hence, with the introduction of this important radiological feature, the p-TACE model was able to predict TACE refractoriness risk accurately before on-demand TACE. The underlying mechanism of the correlations among heterogeneous vascularization, poor tumor differentiation and refractoriness to TACE needs further exploration.

There are some limitations in the present study. Firstly, given that the majority of patients included in this study were patients with HBV-related HCC, the prediction efficacy of p-TACE model might not be consistent for Western patients with HCV-related HCC. Secondly, although the p-TACE scoring model was derived and internally validated on a larger cohort from an Asian liver cancer center, external validation in different regions is necessary. Thirdly, although molecular target therapy may be potentially effective for HCC patients with TACE refractory status, the optimal treatment modality has not been determined in present study. However, the strength of our study lies in: 1) employing accessible clinical parameters and developing an easy-to-use model for clinical application; 2) including a radiological factor of vascularization and assessing tumor features in a visualized manner; 3) identifying pre-treatment TACE refractoriness risk and facilitating early treatment migration. To the best of our knowledge, the p-TACE model is the first pre-treatment model for predicting TACE refractoriness risk in early/intermediate stage HCC.

In conclusion, we successfully derived and validated a pre-treatment scoring model, exhibiting adequate performance for individual prediction of TACE refractoriness in early/intermediate-stage HCC patients following TACE treatment. Further validation of the novel scoring model based on prospective cohorts are warranted.

\section{Abbreviations}

HCC: Hepatocellular carcinoma; HBV: hepatitis $B$ virus; TACE: transcatheter arterial chemoembolization; VEGF: vascular endothelial growth factor; HAP: hepatoma arterial-embolization prognostic; ART: Assessment for Retreatment with TACE; CT: computed tomography; MRI: magnetic resonance imaging; mRECIST: modified response evaluation criteria in solid tumors; NLR: neutrophil-to- lymphocyte ratio; TRIPOD: transparent reporting of a multivariable prediction model for individual prognosis or diagnosis; ROC: receiver operating characteristic curve; AUROC: area under receiver operating characteristic curve; H-L test: Hosmer-Lemeshow test; OR: odds ratio; AFP: alpha-fetoprotein; $\gamma$-GT: $\gamma$-glutamyl transpeptidase; $\mathrm{CI}$ : confidence interval.

\section{Supplementary Material}

Supplementary figures and tables. http://www.jcancer.org/v11p4589s1.pdf

\section{Acknowledgements}

The study was supported by the National Natural Science Foundation of China (No.81672331 and No.81972889).

\section{Competing Interests}

The authors have declared that no competing interest exists.

\section{References}

1. Torre LA, Bray F, Siegel RL, Ferlay J, Lortet-Tieulent J, Jemal A. Global cancer statistics, 2012. CA Cancer J Clin. 2015; 65: 87-108.

2. Yang JD, Hainaut P, Gores GJ, Amadou A, Plymoth A, Roberts LR. A global view of hepatocellular carcinoma: trends, risk, prevention and management. Nat Rev Gastroenterol Hepatol. 2019; 16: 589-604.

3. Bruix J, Reig M, Sherman M. Evidence-Based Diagnosis, Staging, and Treatment of Patients With Hepatocellular Carcinoma. Gastroenterology. 2016; 150: 835-53.

4. Kudo M, Matsui O, Izumi N, Kadoya M, Okusaka T, Miyayama S, et al. Transarterial chemoembolization failure/refractoriness: JSH-LCSGJ criteria 2014 update. Oncology. 2014; 87 Suppl 1: 22-31.

5. Wang B, Xu H, Gao ZQ, Ning HF, Sun YQ, Cao GW. Increased expression of vascular endothelial growth factor in hepatocellular carcinoma after transcatheter arterial chemoembolization. Acta Radiol. 2008; 49: 523-9.

6. Kudo M, Izumi N, Kokudo N, Matsui O, Sakamoto M, Nakashima O, et al. Management of hepatocellular carcinoma in Japan: Consensus-Based Clinical Practice Guidelines proposed by the Japan Society of Hepatology (JSH) 2010 updated version. Dig Dis. 2011; 29: 339-64.

7. Kudo M, Ueshima K. Positioning of a molecular-targeted agent, sorafenib, in the treatment algorithm for hepatocellular carcinoma and implication of many complete remission cases in Japan. Oncology. 2010; 78 Suppl 1: 154-66.

8. Waghray A, Murali AR, Menon KN. Hepatocellular carcinoma: From diagnosis to treatment. World J Hepatol. 2015; 7: 1020-9.

9. Kambhampati S, Bauer KE, Bracci PM, Keenan BP, Behr SC, Gordan JD, et al. Nivolumab in patients with advanced hepatocellular carcinoma and Child-Pugh class B cirrhosis: Safety and clinical outcomes in a retrospective case series. Cancer. 2019; 125: 3234-41.

10. Galle PR, Forner A, Llovet JM, Mazzaferro V, Piscaglia F, Raoul J-L, et al. EASL Clinical Practice Guidelines: Management of hepatocellular carcinoma. J Hepatol. 2018; 69: 182-236.

11. Reig M, Darnell A, Forner A, Rimola J, Ayuso C, Bruix J. Systemic therapy for hepatocellular carcinoma: the issue of treatment stage migration and registration of progression using the BCLC-refined RECIST. Semin Liver Dis. 2014; 34: 444-55.

12. Sangro B, Salem R. Transarterial chemoembolization and radioembolization. Semin Liver Dis. 2014; 34: 435-43.

13. Kadalayil L, Benini R, Pallan L, O'Beirne J, Marelli L, Yu D, et al. A simple prognostic scoring system for patients receiving transarterial embolisation for hepatocellular cancer. Ann Oncol. 2013; 24: 2565-70.

14. Cappelli A, Cucchetti A, Cabibbo G, Mosconi C, Maida M, Attardo S, et al. Refining prognosis after trans-arterial chemo-embolization for hepatocellular carcinoma. Liver Int. 2016; 36: 729-36.

15. Sieghart W, Hucke F, Pinter M, Graziadei I, Vogel W, Muller C, et al. The ART of decision making: retreatment with transarterial chemoembolization in patients with hepatocellular carcinoma. Hepatology. 2013; 57: 2261-73.

16. Kudo M, Arizumi T, Ueshima K. Assessment for retreatment (ART) score for repeated transarterial chemoembolization in patients with hepatocellular carcinoma. Hepatology. 2014; 59: 2424-5. 
17. Terzi E, Terenzi L, Venerandi L, Croci L, Renzulli M, Mosconi C, et al. The ART score is not effective to select patients for transarterial chemoembolization retreatment in an Italian series. Dig Dis. 2014; 32: 711-6.

18. Heimbach JK, Kulik LM, Finn RS, Sirlin CB, Abecassis MM, Roberts LR, et al. AASLD guidelines for the treatment of hepatocellular carcinoma. Hepatology. 2018; 67: 358-80.

19. Yin $\mathrm{X}$, Zhang $\mathrm{L}$, Wang $\mathrm{YH}$, Zhang BH, Gan $\mathrm{YH}$, Ge NL, et al. Transcatheter arterial chemoembolization combined with radiofrequency ablation delays tumor progression and prolongs overall survival in patients with intermediate (BCLC B) hepatocellular carcinoma. BMC Cancer. 2014; 14: 849.

20. Terzi E, Golfieri R, Piscaglia F, Galassi M, Dazzi A, Leoni S, et al. Response rate and clinical outcome of HCC after first and repeated cTACE performed "on demand". J Hepatol. 2012; 57: 1258-67.

21. Johnson PJ, Berhane S, Kagebayashi C, Satomura S, Teng M, Reeves HL, et al. Assessment of liver function in patients with hepatocellular carcinoma: a new evidence-based approach-the ALBI grade. J Clin Oncol. 2015; 33: 550-8.

22. Kawamura $Y$, Ikeda $K$, Hirakawa M, Yatsuji H, Sezaki H, Hosaka T, et al. New classification of dynamic computed tomography images predictive of malignant characteristics of hepatocellular carcinoma. Hepatol Res. 2010; 40: 1006-14.

23. Kawamura $Y$, Ikeda $K$, Seko $Y$, Hosaka T, Kobayashi M, Saitoh S, et al. Heterogeneous type 4 enhancement of hepatocellular carcinoma on dynamic $\mathrm{CT}$ is associated with tumor recurrence after radiofrequency ablation. AJR Am J Roentgenol. 2011; 197: W665-73.

24. Collins GS, Reitsma JB, Altman DG, Moons KG. Transparent reporting of a multivariable prediction model for individual prognosis or diagnosis (TRIPOD): the TRIPOD statement. Bmj. 2015; 350: g7594.

25. Lee SK, Song MJ, Kim SH, Park M. Comparing various scoring system for predicting overall survival according to treatment modalities in hepatocellular carcinoma focused on Platelet-albumin-bilirubin (PALBI) and albumin-bilirubin (ALBI) grade: A nationwide cohort study. PLoS One. 2019; 14: e0216173.

26. Ho CHM, Chiang CL, Lee FAS, Choi HCW, Chan JCH, Yeung CSY, et al. Comparison of platelet-albumin-bilirubin (PALBI), albumin-bilirubin (ALBI), and child-pugh (CP) score for predicting of survival in advanced hcc patients receiving radiotherapy (RT). Oncotarget. 2018; 9: 28818-29.

27. Shan Q, Chen J, Zhang T, Yan R, Wu J, Shu Y, et al. Evaluating histologic differentiation of hepatitis $\mathrm{B}$ virus-related hepatocellular carcinoma using intravoxel incoherent motion and AFP levels alone and in combination. Abdom Radiol (NY). 2017; 42: 2079-88.

28. $\mathrm{Ma} \mathrm{H}$, Zhang $\mathrm{L}$, Tang $\mathrm{B}$, Wang $\mathrm{Y}$, Chen $\mathrm{R}$, Zhang $\mathrm{B}$, et al. gamma-Glutamyltranspeptidase is a prognostic marker of survival and recurrence in radiofrequency-ablation treatment of hepatocellular carcinoma. Ann Surg Oncol. 2014; 21: 3084-9.

29. Peck-Radosavljevic M, Kudo M, Raoul JL, Lee HC, Decaens T, Heo J, et al. Outcomes of Patients (pts) with Hepatocellular Carcinoma (HCC) Treated with Transarterial Chemoembolization (TACE): Global OPTIMIS Final Analysis. Hepatology. 2018; 68: 167A-8A.

30. Kudo M, Ueshima K, Torimura T, Tanabe N, Ikeda M, Aikata H. Randomized, open label, multicenter, phase II trial of transcatheter arterial chemoembolization (TACE) therapy in combination with sorafenib as compared with TACE alone in patients with hepatocellular carcinoma: TACTICS trial. Journal of Clinical Oncology. 2018; 36: 1.

31. Vogel A, Cervantes A, Chau I, Daniele B, Llovet J, Meyer T, et al. Hepatocellular carcinoma: ESMO Clinical Practice Guidelines for diagnosis, treatment and follow-up. Ann Oncol. 2018; 29: iv238-iv55.

32. Reis SP, Sutphin PD, Singal AG, Grzybowski R, Fisher S, Ball C, et al. Tumor Enhancement and Heterogeneity Are Associated With Treatment Response to Drug-Eluting Bead Chemoembolization for Hepatocellular Carcinoma. J Comput Assist Tomogr. 2017; 41: 289-93

33. Chen CS, Li FK, Guo CY, Xiao JC, Hu HT, Cheng HT, et al. Tumor vascularity and lipiodol deposition as early radiological markers for predicting risk of disease progression in patients with unresectable hepatocellular carcinoma after transarterial chemoembolization. Oncotarget. 2016; 7: 7241-52.

34. Nakachi K, Tamai H, Mori $Y$, Shingaki N, Moribata K, Deguchi $H$, et al. Prediction of poorly differentiated hepatocellular carcinoma using contrast computed tomography. Cancer Imaging. 2014; $14: 7$.

35. Pez F, Gifu P, Degli-Esposti D, Fares N, Lopez A, Lefrancois L, et al. In vitro transformation of primary human hepatocytes: Epigenetic changes and stemness properties. Exp Cell Res. 2019: 111643.

36. Rhee H, Nahm JH, Kim H, Choi GH, Yoo JE, Lee HS, et al. Poor outcome of hepatocellular carcinoma with stemness marker under hypoxia: resistance to transarterial chemoembolization. Mod Pathol. 2016; 29: 1038-49. 